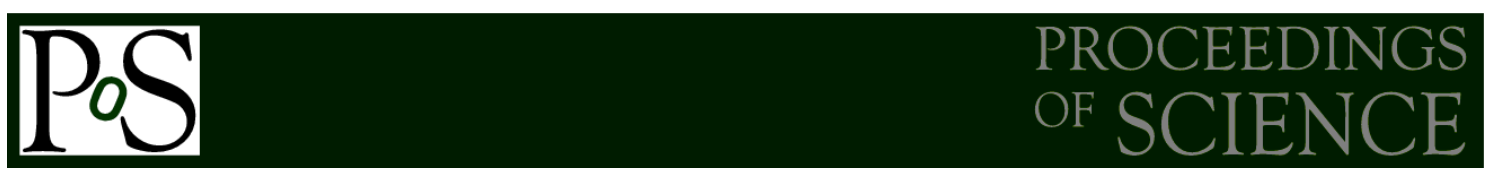

\title{
Re-examination of the First Five Ground-Level Events
}

\author{
M. A. Shea ${ }^{1}$ \\ SSSRC \\ 100 Tennyson Avenue, Nashua, NH 03062, USA \\ E-mail:sssrc@msn.com \\ D. F. Smart \\ SSSRC \\ 100 Tennyson Avenue, Nashua, NH 02062, USA \\ E-mail:donsmart100@msn.com
}

\begin{abstract}
A re-examination of the first five Ground-Level Events (GLEs) has resulted in identification of characteristics that were not noted at the time of the initial publications. These five events, taken collectively, have not been reproduced by the sun since their occurrence primarily because of their intensity at energies $>4 \mathrm{GeV}$ as detected by ionization chambers. While we know that the GLE on 23 February 1956, the largest event to date, was recorded by equatorial cosmic ray detectors, examination of the Huancayo bi-hourly ionization data for the GLEs on 7 March 1942 and 19 November 1949 indicates that these events were also recorded near the equatorial region. In a study of the GLE on 23 February 1956 it was possible to obtain one minute time resolution of the cosmic ray intensity at Huancayo during this event. It should be possible to obtain fine resolution of the timing of the earlier events utilizing the original analog records.
\end{abstract}

36th International Cosmic Ray Conference -ICRC2019-

July 24th - August 1st, 2019

Madison, WI, U.S.A.

\section{${ }^{1}$ M. A. Shea Speaker}

(C) Copyright owned by the author(s) under the terms of the Creative Commons 


\section{Introduction}

The first two confirmed solar proton events occurred a week apart in 1942. These two events were detected as "increases in the cosmic ray intensity" as recorded by the standardized ComptonBennett ionization chambers operated by Scott Forbush but were not identified as increases associated with solar activity [1]. It was not until the event on 25 July 1946 that Forbush suggested that the three "unusual increases" may have been caused by charged particles being emitted by the Sun [2]. All three events were recorded by three of Forbush's ionization chambers: Godhavn, Greenland; Cheltenham, USA; Christchurch, New Zealand. The event on 25 July 1946 was also recorded by an ionization chamber in Manchester, UK [3]. At that period in cosmic ray history there were serious questions as to whether the sun could emit solar particles energetic enough to be recorded by detectors at Earth, with distinguished scientists such as Hannes Alfvén stating "that the variations in cosmic radiation are due to changes in the earth's electrostatic potential" [4].

After a presentation on the early relativistic solar proton events at the COSPAR meeting in Montreal in 2008, we were approached by Dr. Jun Nishimura who informed us that the event on 7 March 1942 had also been recorded by five ionization chambers of the Nishina Laboratory in Tokyo. While this increase was reported at a meeting of the Riken at that time, the increase was so unusual it was believed to be caused by noise on the electrical power which was unstable during this war time period. Since the geomagnetic cutoff rigidity of Tokyo was approximately 11.35 GV in 1942, this event was apparently much more energetic than previously realized. In the articles by Forbush, he specifically stated that "no such increase took place at Huancayo" $[1,5]$. The increase in Japan prompted us to revisit the history and historical records associated with the first five ground-level solar cosmic ray events.

Table 1. Solar Activity Associated with the First Five GLEs

$\begin{array}{llllll}\begin{array}{l}\text { GLE } \\ \text { No. }\end{array} & \text { Date } & \begin{array}{c}\text { Solar } \\ \text { Coordinates }\end{array} & \begin{array}{l}\text { H alpha } \\ \text { Onset (UT) }\end{array} & \begin{array}{l}\text { Radio } \\ \text { Onset (UT) }\end{array} & \text { Major GMS } \\ 1 & \text { 28 Feb 1942 } & \text { 07N, 04E } & \text { N.O. } & 1200 & \text { Yes; Kp = 8 } \\ 2 & \text { 7 Mar 1942 } & \text { 07N, 90W } & \text { N.O. } & 0442 & \text { No } \\ 3 & \text { 25 Jul 1946 } & \text { 22N, 15E } & 1615 & 1634 & \text { Yes; Kp =9 } \\ 4 & \text { 19 Nov 1949 } & \text { 02S, 70W } & 1029 & 1029 & \text { No } \\ 5 & \text { 23 Feb 1956 } & \text { 23N, 80W } & \text { 0331 } & 0332 & \text { Yes; see Notes }\end{array}$

Notes: N.O. $=$ No observation

A major GMS occurred on 25 February with $\mathrm{Kp}=8+$. The source is uncertain.

Table 1 presents a summary of the solar activity associated with the first five Ground-Level Events (GLEs). Solar activity near the central meridian of the sun was associated with the GLEs on 28 February 1942 and 25 July 1946; the solar activity associated with the remaining three events was near or at the western limb of the solar disk. Magnetic crochets were recorded during each of these flares indicative of a powerful impulsive X-ray event [6]. Studies of high energy solar proton events show that particle increases at the earth from central meridian solar activity typically have a slower rise time and softer spectra than particle increases from solar activity near, 
at or slightly behind the western limb of the sun [7]. In addition major solar activity from the solar central meridian is typically associated with a major geomagnetic storm a day or two later when the coronal mass ejection, which propagates in the radial direction, reaches the earth. Four of these GLEs followed the typical pattern. Event number 5, 23 February 1956 has the highest increase to date (based on 15-minute averages). While a major geomagnetic storm (GMS) occurred on 25 February 1956, it is not certain that the solar activity on 23 February was the source of this geomagnetic disturbance.

\section{Cosmic Ray Observations}

The detection of high energy solar particles on the earth is a function of the type of detector, the geomagnetic cutoff rigidity of the station location, and the direction in space at which the detector is viewing at the time of the event. Ionization chambers respond to incident energetic particles greater than $\sim 4 \mathrm{GeV}$. Stations at high geomagnetic cutoff rigidities would respond only to those particles having rigidities higher than the station geomagnetic cutoff. In 1942 Huancayo had a vertical geomagnetic cutoff rigidity of $\sim 13.64 \mathrm{GV}$. Each location on the earth views a region in space as the earth rotates. This region is called the asymptotic cone of acceptance. Since high energy particles emitted from the solar active region typically follow the interplanetary field lines in space, a station viewing into the direction of the interplanetary field during the onset of an energetic solar particle event typically would record an appropriate increase above the background cosmic ray intensity. Figure 1 illustrates the basic asymptotic cones of acceptance for the four Forbush ionization chambers and for a detector at Tokyo, Japan. While the cones for Godhavn, Cheltenham and Christchurch are "relatively narrow", locations at high cutoff rigidity values such as Tokyo and Huancayo have cones that extend over a large range of longitudes.
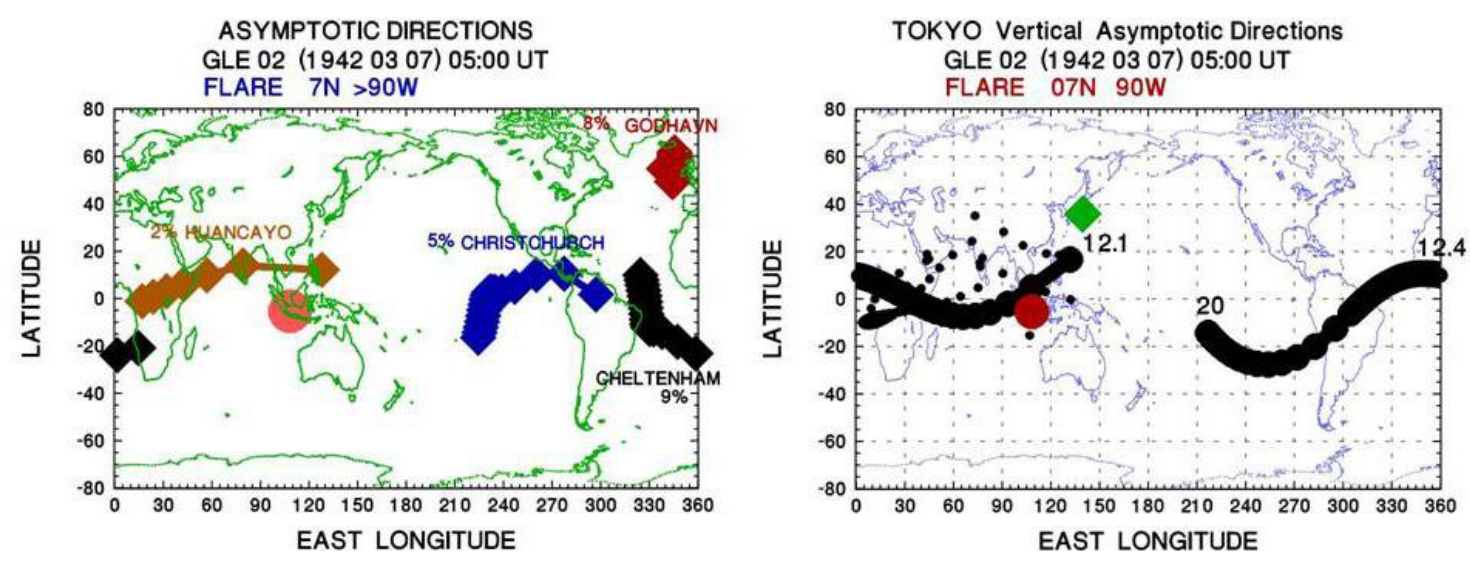

Figure 1. Left Side: Asymptotic cones for the Forbush ionizations chambers appropriate for the GLE on 7 March 1942. Right Side: Asymptotic cone for the ionization chambers in Tokyo for the same time period. The smaller circles scatted above the main cone in the Tokyo plot are allowed particles in the cosmic ray penumbra. The red dot at longitude $105^{\circ} \mathrm{E}$ is the sub-solar point at the time of the solar radio burst. 


\section{Available Data and Discussion}

The Carnegie Institution published a series of data books $[8,9,10,11]$ containing data from the Forbush ionization chambers. Table 1 in Shea and Smart [12] details the data and time intervals included in these publications. Unfortunately the only bi-hourly data available in these publications for the first four GLEs are from Cheltenham and Huancayo. Data from these two stations as well as from the Godhavn detector were published for GLE 5 (23 February 1956). Forbush also operated identical Compton-Bennett ionization chambers in Teoloyucan, Mexico and Climax, Colorado during part of this time period; however, these data were not included in these four Carnegie publications. From Figure 1 in Forbush et al. [5] no increase was seen at Teoloyucan, Mexico for the event on 28 February 1942; the station apparently had operational problems shortly thereafter and was not operating for the subsequent GLE on 7 March 1942.

CHELTENHAM IONIZATION CHAMBER

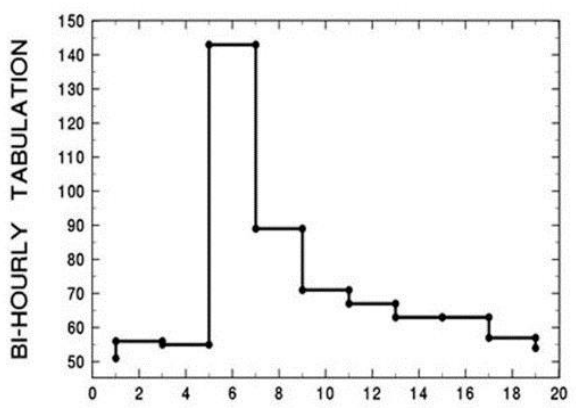

UT HOUR 7 March 1942
HUANCAYO IONIZATION CHAMBER

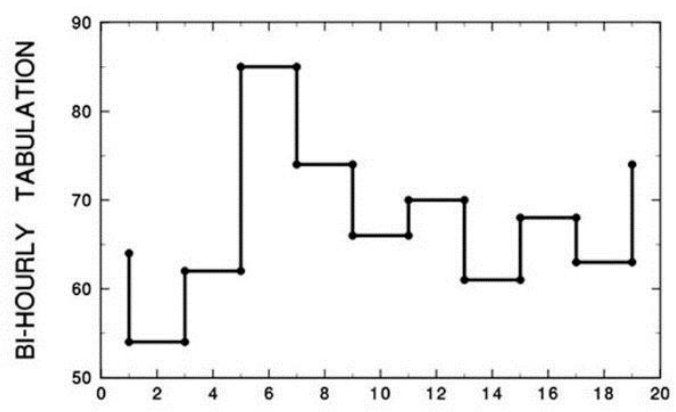

UT HOUR 7 March 1942

Figure 2. Comparison of Cheltenham and Huancayo data for 7 March 1942. Left side: Cheltenham bi-hourly mean values of departures from balance, +100 , in units of $0.1 \%$ of the total cosmic-ray ionization. Right side: Huancayo bi-hourly mean values of departure from balance, +60 in units of $0.1 \%$ of the total cosmic-ray ionization. Data are corrected for local mean atmospheric pressure. The solar radio onset was 0442 UT.

From an examination of the bi-hourly data from Cheltenham and Huancayo for the two events associated with central meridian solar activity (28 Feb 1942, 24 July 1946) no increase is evident in the Huancayo data. In a comparison of the data for these two stations for the event on 7 March 1942, a slight increase above the background cosmic ray intensity is evident at Huancayo coincident with the increase at Cheltenham as shown in Figure 2. Careful inspection of Figure 3 in [1] shows a coincident increase at Huancayo. This time coincident increase was identified as a $1 \%$ increase by Ellison et al. [13]. Hourly data from four ionization chambers operating in Japan, one of which was underground, also reveal simultaneous increases in the 0400-0600 UT time period [14]. Assuming that the interplanetary magnetic field direction is along a direction $\sim 45$ degrees west of the sub-solar longitude ( $\sim 60^{\circ}$ E geographic longitude on Figure 1$)$, both the Tokyo and Hunacayo detectors were viewing in approximately the same direction in space. If the solar particle flux contained particles with rigidity values above the cutoff rigidity of Huancayo, it would appear likely that the Huancayo ionization chamber might also record an increase in cosmic ray particles at this time. Other ionization chambers were known to have recorded the 
events in $1942[15,16]$ but detailed data are not available to us. While there was an ionization chamber operating on Hafelekar, Austria, from 1933 onward, it is not known if any unusual increases were observed or if the data were archived for future use.

The GLE on 19 November 1949 was recorded by several cosmic ray detectors including five muon detectors and an experimental neutron monitor. The neutron monitor, located in Manchester, UK, recorded an increase of $550 \%$ (hourly data) above the previous background intensity [17]. The Cheltenham ionization chamber recorded an increase of $41 \%$ in bi-hourly data whereas the Compton-Bennett ionization chamber recently installed at Climax, Colorado, USA (altitude 3500 meters) recorded an increase of $\sim 180 \%$ in a 15 -minute time interval [5]. The detector at Climax was under additional shielding with Forbush et al. [5] estimating that the increase would have been $\sim 207 \%$ without the shielding. (Note: the values given in [5] were from uncorrected data.) The onset of the event at Climax was 1045 UT; the event continued for several hours. An inspection of the Cheltenham and Huancayo bi-hourly data [8,9] again reveals a slight increase at Huancayo as shown in Figure 3. Assuming the onset of the event at Huancayo was similar to that at Climax, the event clearly continued into the next bi-hourly segment. The muon detector at Bagneres, France (cutoff rigidity 5.4 GV) recorded a $4 \%$ increase during the November 1949 GLE. We do not know if there were muon or ionization chamber data available for stations with a higher geomagnetic cutoff rigidity during this event to verify the slight increase at Huancayo as an equatorial observed GLE.
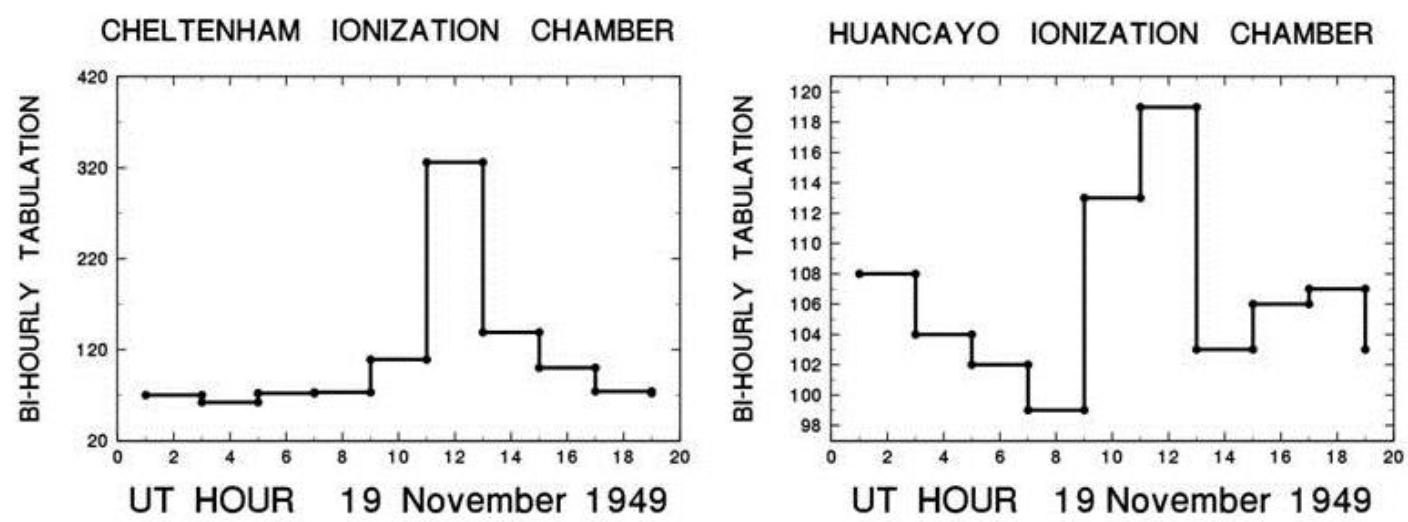

Figure 3. Comparison of Cheltenham and Huancayo ionization chamber data for 19 November 1949. Left side: Cheltenham bi-hourly mean values of departures from balance, + 100 , in units of $0.1 \%$ of the total cosmic-ray ionization. Right side: Huancayo bi-hourly mean values of departure from balance, +60 in units of $0.1 \%$ of the total cosmic-ray ionization. Data are corrected for local mean atmospheric pressure. H-alpha flare onset was 1029 UT.

In July 2004, Dr. K. G. McCracken inspected 32 boxes containing the entire Forbush records which were stored at the Carnegie Institution in Washington, DC. Subsequently eleven of these boxes were transferred to the US National Geophysical Data Center in Boulder, Colorado where they have been partially scanned for archival purposes. Table 2 gives a summary of the analog data that were transferred. The station log books, sample hourly data and scaling details were also included. These original analog records can be differentiated to obtain high-time resolution 
for each of the GLEs for which recordings are available. An examination of the detailed ionization traces preserved by Scott Forbush has enabled a resolution of the onset time of the 23 February 1956 event at Huancayo to within 15 seconds [18].

Table 2. Forbush data archives transferred to the National Geophysical Data Center

Huancayo photographic records, 1936-1960

Cheltenham/Fredericksburg photographic records, 1935-1960

Christchurch photographic records, 1936-1959

Mexico photographic records; 1954-1959

Godhavn photographic records, 1938-1959

Climax photograph records, 1949-1959

The five high energy solar proton events between 1942 and February 1956 are unique in the history of cosmic ray physics. Examination of the Forbush analog records could provide accurate onsets of the observed increases as well as the intensity/time profiles throughout the event. The $20 \%$ increase in the ionization chamber bi-hourly measurement for the 25 July 1946 event is the highest increase recorded by an ionization chamber or muon detector from a central meridian solar event.

The possibility of identifying an earlier ground-level event is intriguing. The Forbush analog data cover a time period earlier than tabulated in the Carnegie Institution publications. From a study of ionospheric data, Svestka [19] has compiled a list of possible proton flares from 1938-1955 which could be investigated. Beer and McCracken [20] have found impulsive enhancements in the ${ }^{10} \mathrm{Be}$ records approximately 2 years following a known ground-level event, and list one enhancement in 1938 which they suggest might be associated with the solar activity in April 1937. Data for Cheltenham, Christchurch and Huancayo are available for that time period. Researching data immediately following a strong magnetic crochet, particularly associated with a flare near the western disk of the sun would be another avenue to explore.

\section{Summary}

Identification of small increases at Huancayo for the GLEs on 7 March 1942 and 19 November 1949 show that these two events were more energetic than previously realized. To our knowledge, the increase at an underground ionization chamber in Japan on 7 March 1942 is the only increase from solar particles recorded by an underground detector at high cutoff rigidities. The GLE on 29 September was not recorded by the underground detector in Japan [21]. The possibility of obtaining intensity/time profiles with minute resolution from the Forbush archived data would provide a new understanding of these early events and should be investigated by experts who can correctly interpret these analog records. Finally, if additional ionization chamber or muon data for the first five events are still available and not permanently archived, they should be catalogued for future research.

Acknowledgements. The authors greatly appreciate the documents provided by Dr. Jun Nishimura on the ionization chamber measurements in Japan in 1942. 


\section{References}

[1] I. Lange and S. E. Forbush, Further Note on the Effect on Cosmic-Ray Intensity of the Magnetic Storm of March 1, 1942, Terr. Mag. 47 (1942) 331.

[2] S. E. Forbush, Three Unusual Cosmic-Ray Increases Possibly Due to Charged Particles from the Sun, Phys. Rev. 70 (1946) 771.

[3] D.W.N. Dolbear and H. Elliot, Cosmic Ray Intensity at Manchester and Solar Disturbances of July 25, 1946, Nature 159 (1947) 58.

[4] H. Alfvén, Changes in Cosmic Ray Intensity Associated with Magnetic Storms, Nature 158 (1946) 158.

[5] S.E. Forbush et al., The Extraordinary Increase of Cosmic-Ray Intensity on November 19, 1949, Phys. Rev. 79 (1950) 501.

[6] S-I. Akasofu and S. Chapman in Solar-Terrestrial Physics, Oxford University Press, London, pg. 522 (1972).

[7] M.A. Shea and D.F. Smart, History of Energetic Solar Protons for the Past Three Solar Cycles including Cycle 22 Update, in Biological Effects and Physics of Solar and Galactic Cosmic Radiation, Part B, Edited by Charles E. Swenberg, Gerda Horneck, and E.G. Stassinopoulos, 37-71, Plenum Press, New York (1993).

[8] I. Lange and S.E. Forbush, Cosmic-Ray Results from Huancayo Observatory, Peru, June, 1936-December, 1946, Researches of the Department of Terrestrial Magnetism XIV, Carnegie Institution of Washington Publication 175 (1948).

[9] I. Lange and S.E. Forbush, Cosmic-Ray Results, Researches of the Department of Terrestrial Magnetism XX, Carnegie Institution of Washington Publication 175 (1957).

[10] L. Beach and S.E. Forbush, Cosmic-Ray Results, Researches of the Department of Terrestrial Magnetism XXI, Carnegie Institution of Washington Publication 175 (1961).

[11] L. Beach, and S. E. Forbush, Cosmic-Ray Results, Researches of the Department of Terrestrial Magnetism XXII, Carnegie Institution of Washington Publication 175 (1969).

[12] M.A. Shea and D.F. Smart, Fifty Years of Cosmic Radiation Data, Space Sci. Rev. 93 (2000) 229-262.

[13] M.A. Ellison, S.M.P. McKenna and J.H. Reid, Cosmic Ray Flares, Dunsink Observatory Publications 1 (1961).

[14] J. Nishimura, Historical Aspect for the Development of High Energy Astrophysics in Japan, Presentation, International Workshop on Cosmic rays and High Energy Universe, Tokyo (2007).

[15] E. B. Berry and V.F. Hess, Study of Cosmic Rays Between New York and Chile, Terr. Mag. 47 (1942) 251.

[16] J. Firor, Cosmic Radiation Intensity-Time Variations and Their Origin, IV. Increases Associated with Solar Flares, Phys. Rev. 94, 1017 (1954).

[17] N. Adams, A Temporary Increase in the Neutron Component of Cosmic Rays, Phil. Mag. 41 (1950) 503. 
[18] K. McCracken, M.A. Shea and D. Smart, The Short-lived ( $<2$ minutes) Acceleration of Protons to $>13 \mathrm{GeV}$ in Association with Solar Flares, Presentation at EGU, April (2016).

[19] Z. Švestka, Proton Flares Before 1956, BAC 17 (1966) 262.

[20] K.G. McCracken and J. Beer, The Annual Cosmic-Radiation Intensities 1391-2014; The Annual Heliospheric Magnetic Field Strengths 1391 - 1983, and Identification of Solar Cosmic-Ray Events in the Cosmogenic Record 1800 - 1983, Sol. Phys, 290 (2015) 3051.

[21] Swinson, D.B., and M.A. Shea, The September 29, 1989 Ground-Level Event Observed at High Rigidity, Geophys. Res. Letters 17 (1990) 1073. 